\title{
PSA-based screening in Sweden dramatically reduces disease-specific mortality
}

A fter 14 years of follow-up, data from the Göteborg trial in Sweden show that PSA-based screening is associated with a halving of deaths due to prostate cancer.

The Göteborg study, which included 20,000 randomly selected men, adds weight to the main finding of the European Randomised Study of Screening for Prostate Cancer (ERSPC). Last year, ERSPC investigators determined that their population-based screening program also reduced the rate of prostate-cancer-related death.

The Göteborg protocol involved prospective randomization of Swedish residents to either a 10,000-strong screening cohort or a control group of equal size. Men allocated to screening were offered a PSA test every 2 years. Three-quarters attended at least one round of screening.

Almost 30,000 PSA tests were performed. The PSA concentration that triggered further workup dropped slightly during the study period, from $3.4 \mathrm{ng} / \mathrm{ml}$ to $2.5 \mathrm{ng} / \mathrm{ml}$. This change primarily reflected alignment of the Göteborg trial

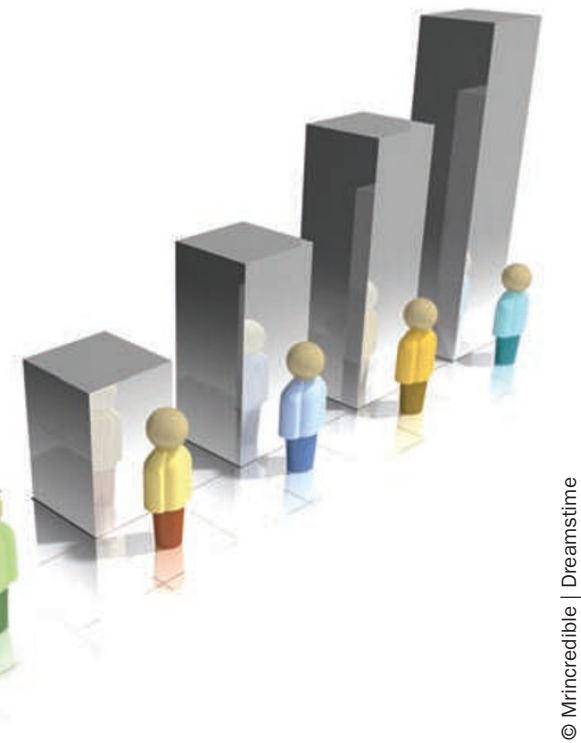

with the ERSPC in 1999. The PSA level of just over a third of those who presented for screening was found to exceed these thresholds.

The workup offered to participants with an elevated PSA level comprised digital rectal examination, transrectal ultrasound, and sextant biopsies. More than $90 \%$ of eligible men elected to undergo these invasive investigations. Just over 4,100 biopsies were performed.

A diagnosis of prostate cancer was made for 1,138 men in the screening group. Approximately $80 \%$ of these diagnoses were made as a direct result of participation in the Göteborg trial. Fewer cases of prostate cancer $(n=718)$ were reported in the control cohort (hazard ratio 1.64).

The relative risk of dying from prostate cancer was 0.56 in the screening group, relative to the control arm. The magnitude of this positive outcome dramatically exceeds that reported for the ERSPC (relative risk 0.80).

In an Editorial that accompanies the Göteborg team's paper in The Lancet Oncology, David Neal from the University of Cambridge in the UK highlights two features of the Göteborg trial that might underlie the discrepancy in main outcome between it and the ERSPC. First, participants were followed up for longer during the Göteborg trial (14 years versus 9 years in the ERSPC). Second, screening began at a younger age.

Lead author on the Göteborg team's paper Jonas Hugosson comments: “Among men who were under 60 years [of age] when they entered the study and who attended [screening], the risk of dying from prostate cancer was only one-quarter [of] that in the control group, indicating that PSA screening-if started early (at 50-55 years of age) - seems to markedly reduce the risk of dying from prostate cancer."

The relative 'naivety' of the Swedish population to PSA testing is another important feature of the Göteborg trial. "The study started in 1995 and, at that time, PSA testing was very infrequent in Sweden and remained [so for] at least the first 5 years of the study", reports Hugosson.

The low level of opportunistic PSA testing kept 'contamination' of the Göteborg control cohort to a minimum. This is in stark contrast to the US-based Prostate, Lung, Colorectal, and Ovarian (PLCO) Cancer Screening Trial, in which up to $50 \%$ of men in the control arm underwent PSA testing or digital rectal examination. The PLCO trial failed to detect any benefit of screening.

The large degree of variation in practice and population characteristics between countries means that study findings should be geographically extrapolated with caution. Neal states that the Göteborg results might be generalizable to other regions that have not had prior extensive PSA testing, but not to countries such as the US, in which testing is widely available.

The Göteborg trialists calculated that 293 men would need to be screened, and 12 treated, to prevent one death from prostate cancer. These numbers, which are markedly lower than those determined by the ERSPC investigators, represent a cost/benefit ratio approximating that of screening programs for breast cancer.

Concerns about overtreatment of prostate cancer might further be allayed by the increasing popularity of active surveillance for early stage disease. "One important finding in [the Göteborg] study is that diagnosis of prostate cancer did not automatically result in men taking up radical treatment", comments Neal.

Suzanne J. Farley

Original article Hugosson, J. et al. Mortality results from the Göteborg randomised population-based prostate-cancer screening trial. Lancet Oncol. doi:10.1016/S1470 2045(10)70146-7 